\title{
Adsorption and Diffusion of Benzene in ITQ-1 Type Zeolite: Grand Canonical Monte Carlo and Molecular Dynamics Simulation Study
}

\author{
T. J. Hou, L. L. Zhu, and X. J. Xu* \\ College of Chemistry and Molecular Engineering, Peking University, \\ Beijing 100871, People's Republic of China \\ Received: February 4, 2000; In Final Form: July 17, 2000
}

\begin{abstract}
The methods of grand canonical Monte Carlo (GCMC) simulation and molecular dynamics (MD) are used for investigating the adsorption and diffusion of benzene in ITQ-1 zeolite. First, the adsorption energies and preferred adsorption sites of benzene in ITQ-1 are determined by using GCMC simulations. The obtained mass clouds show that the diffusion and the mobility of benzene mainly happen in 12-MR supercages where the preferred adsorption sites of benzene can be divided to three separate adsorption sites. Then MD simulations are employed to analyze the dynamic diffusion processes in zeolite. The calculated diffusion coefficients are compared with the results of Sastre et al. (Sastre, G.; Catlow, C.; Corma, A. J. Phys. Chem. 1999, 103, 5187). From the trajectories followed by the benzene molecules, it can be seen that the guest molecules do not diffuse in both channel systems, and only intracage mobility is seen in the supercage voids. Moreover, the two benzene molecules near the S2 and S3 sites prefer to adopt a synergistic state, indicating that the benzene molecules at the S2 and S3 sites can produce relatively strong aromatic stacking interactions. In the present study, two sets of potential parameters are compared. For the molecules studied, these two force fields furnish similar and comparable results, although the obtained interaction energy and adsorption isotherms exhibit some differences.
\end{abstract}

\section{Introduction}

Among the materials of interest for chemical science and technology, zeolites are outstanding for their special characteristics and multifarious and widespread uses. Zeolite is a kind of porous crystalline aluminosilicate built up by corner-sharing $\mathrm{TO}_{4}$ tetrahedra $(\mathrm{T}=\mathrm{Si}$ or $\mathrm{Al}$ ), giving rise to a rather complex but precisely repetitive atomic network with regular cavities joined by channels in which guest molecules of appropriate size can be accommodated. Adsorption phenomenon in zeolitic microporous materials is of great scientific interest due to applications in separation technology and catalysis, but comprehensive theory on zeolite adsorption still remains unavailable. The diffusion of sorbate molecules in zeolites is very complicated, and the intracrystalline diffusivity will be affected by many elements, including the size and shape of the sorbate molecules relative to the zeolite pore, the energetic interactions between zeolite and sorbate molecules, and the connectivity and dimensionality of the zeolite pore structure. Some microscopic and dynamical diffusion processes of sorbate molecules in zeolites are generally very difficult or impossible to be interpreted only by experiments, so it would be highly desirable to predict the localization or diffusion of adsorbate a priori from fundamental knowledge of the structural and energetic levels of a zeolite-adsorbate system.

In the previous decades, many simulation techniques, including molecular mechanics, ${ }^{1,2}$ molecular dynamics, ${ }^{3-8}$ and Monte Carlo simulation, ${ }^{9,10}$ have been applied to explore the mobility and diffusion of sorbate in zeolite cavities. Among these methods, molecular dynamics (MD) and grand canonical Monte Carlo simulation (GCMC) may be the most widely used

* Corresponding author. E-mail: xiaojxu@chemms.chem.pku.edu.cn. techniques. MD gives access to time-dependent properties and has already been used to study the diffusive behaviors of sorbate, while GCMC simulation can predict the zeolite adsorption phase equilibration. The combination of MD and GCMC can afford us the static and dynamic properties of sorbate in zeolites.

Zeolite MCM-22 (IZA code MWW) is a novel zeolite discovered recently by scientists at Mobil. ${ }^{11,12}$ The unusual framework topology, high thermal stability, large surface area, and good sorption capacity render this zeolite very interesting for catalysis. The studies of the diffusion behavior of aromatics in MCM-22 are very important because many important separations and reactions in MCM-22 are concerned with aromatics, ${ }^{13-15}$ including the alkylation of benzene with propylene to produce cumene, the isomerization of dimethyl benzene, and the disproportionation of toluene. So research on the diffusion and the mobility behavior of benzene in MCM22 zeolite will be helpful for interpreting diffusion and other reactions in the zeolite cavities.

In this paper, GCMC simulation and MD are used to explore how sorbate is located within the zeolite and how sorbate diffuses through the 10-MR sinusoidal channels and the 12MR supercages in the MCM-22 type zeolite. All simulations are performed in the Cerius ${ }^{2}$ molecular simulations package ${ }^{16}$ on a SGI 2-CPU octane workstation.

\section{Methodology}

Pure Siliceous ITQ-1 Structure. Compared with other usual types of zeolites, MCM-22 possesses an interesting and unusual framework structure: two independent pore systems formed by interconnected sinusoidal 10-MR pores with a $4 \times 5.5 \AA$ diameter and an independent 12-MR supercage with $18.2 \times$ $7.1 \AA$ A linked by $10-\mathrm{MR}$ windows. These coexisting pore systems 
may provide opportunities for a wide variety of catalytic applications in the petrochemical and refining industries.

It is well-known that without experimental data, it is very difficult to determine the location of $\mathrm{Al}$ in zeolites only by theoretical calculations. In our previous work, a simple and general method of identifying the $\mathrm{Al}$ atom positions in zeolite was proposed. We found that there existed a good linear relationship between $\Delta H_{\mathrm{f}}$ (heat of formation) and SDR (sum of the reciprocal of distance among $\mathrm{Al}$ atoms). ${ }^{17} \mathrm{In}$ that work, the calculation capability of the quantum chemical techniques was considered, but only one basic framework FAU was used. We think that the there still exist some difficulties in applying the proposed model to the location of $\mathrm{Al}$ for a relatively large system, including a complete zeolite framework or a zeolite superlattice. So considering the difficulty in determining $\mathrm{Al}$ distributions in disordered zeolites and the high $\mathrm{Si} / \mathrm{Al}$ ratio of MCM-22, we adopted the pure silica analogue of MCM-22, ITQ-1, in this paper to simplify the simulations. The model of ITQ-1 is constructed according to the results from Camblor et al. ${ }^{18}$ The zeolite structure is described in the $P 6 / \mathrm{mmm}$ space group with $a=14.2081 \AA$ and $c=24.9452 \AA$.

Potential Parameters. With respect to the special characteristics of the zeolite structure, some potential parameters for zeolite may be different from other molecules, for example, organic molecules or macromolecules. From the references, many types of force fields for zeolite are available, but it seems that many of them lack strict validations. So in the simulation of zeolite, it is very important to carefully choose the adequate force field. In this paper, the potential parameters are selected from the CVFF, which has been fully validated by MSI. Moreover, as a comparison, another set of potential parameters from the research work of Burchart and Mayo is applied.

Potential Parameters from the CVFF. The consistentvalence force field (CVFF) is a generalized valence force field developed by Dauber-Osguthorpe in 1988. ${ }^{19,20}$ The original parameters are provided for amino acids, water, and a variety of other functional groups. Then the augmented CVFF is developed for materials science applications and includes additional atom types for aluminosilicates and aluminophosphates.

Using the CVFF, we can fully consider both the covalent and the nonbonding interactions of the system. The potential of the studied system comes from four parts: the potential of benzene and zeolite, the nonbonding interactions between the framework and sorbate, and the nonbonding interactions among sorbate molecules. The general expression for the adsorbatezeolite potential function is given by

$$
V_{\text {total }}=V_{\text {zeolite }}+V_{\text {sorbates }}+V_{\text {zeolite-sorbates }}+V_{\text {sorbates-sorbates }}
$$

The bonding potentials of the framework and the benzene comprise two- (bond), three- (angle), and four-body (dihedral) interactions

$$
\begin{aligned}
& V_{\text {internal }}=V_{\text {two-body }}+V_{\text {three-body }}+V_{\text {four-body }}= \\
& \quad k_{i j}\left(r_{i j}-r_{i j}^{0}\right)^{2}+k_{i j k}\left(\theta_{i j k}-\theta_{i j k}^{0}\right)^{2}+k_{i j k l}\left[1+\cos \left(n \delta_{i j k}-\delta_{i j k}^{0}\right)\right]
\end{aligned}
$$

Four different atom types are considered: oz (O atom in the zeolite framework), sz ( $\mathrm{Si}$ atom in the zeolite framework), $\mathrm{cp}$ ( $\mathrm{C}$ atom in the benzene ring), and $\mathrm{h}$ ( $\mathrm{H}$ atom in the benzene ring). The system includes four kinds of bonds, four types of angles, and three kinds of dihedrals. The potential parameters for the framework and sorbate are listed in Table 1.

\begin{tabular}{|c|c|c|}
\hline two-body parameters & $R_{i j}{ }^{0}(\AA)$ & $K_{i j}\left(\mathrm{ev} \AA^{-2}\right)$ \\
\hline sz-sz & 3.0900 & 392.8000 \\
\hline SZ-oz & 1.6150 & 392.8000 \\
\hline сp-cp & 1.3400 & 480.0000 \\
\hline $\mathrm{cp}-\mathrm{h}$ & 1.0800 & 363.4164 \\
\hline three-body parameters & $\theta(\mathrm{deg})$ & $K_{i j k}(\mathrm{eV})$ \\
\hline SZ-OZ-SZ & 149.8000 & 31.1000 \\
\hline OZ-SZ-OZ & 109.4700 & 100.3000 \\
\hline cp-cp-cp & 120.0000 & 90.0000 \\
\hline cp-cp-h & 120.0000 & 37.0000 \\
\hline four-body parameters & $k_{i j k l}(\mathrm{eV})$ & $\delta_{i j k l}(\operatorname{deg})$ \\
\hline$*_{-\mathrm{SZ}-\mathrm{OZ}-*}$ & 1.0000 & 0.0000 \\
\hline$*_{\text {-sz-sz- }} *$ & 1.0000 & 0.0000 \\
\hline$*_{\text {-cp-cp- }} *$ & 12.0000 & 180.0000 \\
\hline
\end{tabular}

TABLE 1: Bonding Potential Parameters for the Framework and Sorbate

TABLE 2: van der Waals Parameters and Partial Charges for the Framework and Sorbate

\begin{tabular}{crrr}
\hline atom type & \multicolumn{1}{c}{$A\left(\mathrm{eV}^{12}\right)$} & \multicolumn{1}{c}{$B\left(\mathrm{eV}^{6}\right)$} & \multicolumn{1}{c}{$q(|\mathrm{e}|)$} \\
\hline $\mathrm{sz}$ & 3149175.0000 & 710.00000 & -0.3000 \\
$\mathrm{oz}$ & 272894.7846 & 498.87880 & +0.6000 \\
$\mathrm{cp}$ & 1981049.2250 & 1125.99800 & -0.1000 \\
$\mathrm{~h}$ & 7108.4660 & 32.87076 & +0.1000
\end{tabular}

The nonbonding interaction of the system comprises the $V_{\text {zeolite }}$-sorbate between the guest molecules and the zeolite and the $V_{\text {sorbate-sorbate }}$ among the guest molecules, which is described by the sum of a Lennard-Jones potential and a coulomb potential

$$
V_{\text {nonbond }}=R_{\mathrm{vdW}}+E_{\text {electrostatic }}=\frac{A_{i j}}{R_{i j}{ }^{12}}-\frac{B_{i j}}{R_{i j}{ }^{6}}+\frac{q_{i} q_{j}}{R_{i j}}
$$

where $i$ and $j$ indicate atoms of the sorbate molecule and those of the zeolite, respectively, and $R_{i j}$ is the distance between them. $A_{i j}$ and $B_{i j}$ are the Lennard-Jones parameters, while $q_{i}$ or $q_{j}$ is the partial charge of the atoms. The parameters $A_{i j}$ and $B_{i j}$ are computed by the van der Waals parameters of the pair atoms (Table 2) by geometric mean. The van der Waals parameters for every atom are listed in Table 2, and the partial charges of the atoms in the system are also derived from bond increment parameters in the CVFF.

Potential Parameters from the Burchart-Dreiding Force Field. The Burchart force field was developed by Burchart to describe the geometries, heats of formation, transitions under pressure, crystal morphologies, and vibrational frequencies of silicas and aluminophosphates. ${ }^{21}$ The parametrization is based mainly on experimental data and includes both valence and nonbonding terms. It contains four atom types: $\mathrm{Si}, \mathrm{O}, \mathrm{Al}$, and P. The Dreiding force field, developed by Mayo et al., ${ }^{22}$ is a good, all-purpose force field that can be used for structure predictions and dynamics calculations of organic, biological, and main-group inorganic molecules. The Burchart-Dreiding force field combines the Burchart and Dreiding force fields. The Burchart force field treats the framework, and the Dreiding force field treats the intra- and intermolecular interactions. The parameters for the framework-molecule interactions are derived from the parameters of both force fields, in conjunction with the arithmetic combination rule.

In this paper, the Burchart-Dreiding force field is applied to the GCMC simulations, and nonbonding interactions between the sorbate molecules and the zeolite framework are assumed to interact via a pairwise-additive potential between the atoms of the adsorbed molecules and those of the zeolite. The 
TABLE 3: Lennard-Jones Parameter and Partial Charges for Four Types of Atoms

\begin{tabular}{cccc}
\hline atom type & $D_{0}(\mathrm{kcal} / \mathrm{mol})$ & $R_{0}(\AA)$ & $q(|\mathrm{e}|)$ \\
\hline O_Z & 0.1648 & 3.3000 & -0.1900 \\
Si_Z & 0.0496 & 4.2000 & +0.3800 \\
C_R & 0.0951 & 3.8983 & -0.1300 \\
H & 0.0152 & 3.1950 & +0.1300
\end{tabular}

site-site interactions are modeled with Lennard-Jones and coulomb potentials

$$
V\left(r_{i j}\right)=D_{i j}\left\{\left[\frac{\left(R_{0}\right)_{i j}}{R_{i j}}\right]^{12}-2\left[\frac{\left(R_{0}\right)_{i j}}{R_{i j}}\right]^{6}\right\}+\frac{q_{i} q_{j}}{R_{i j}}
$$

where $i$ and $j$ indicate the atoms of the sorbate and those of the zeolite, respectively, and $R_{i j}$ is the distance between them. $D_{i j}$ and $\left(R_{0}\right)_{i j}$ are the Lennard-Jones parameters, and $q_{i}$ and $q_{j}$ are the partial charges of the atoms. Four different atom types are considered in the system, including $\mathrm{O} \_\mathrm{z}(\mathrm{O}$ atom in the zeolite framework), $\mathrm{Si}$ Z (Si atom in the zeolite framework), C_R (C atom in the benzene ring), and $\mathrm{H}$ ( $\mathrm{H}$ atom in the benzene ring). The Lennard-Jones parameters for $\mathrm{O} \_\mathrm{Z}$ and $\mathrm{Si} \_\mathrm{Z}$ originally derived by Burchart ${ }^{20}$ and those for other two atom types taken from Mayo $^{21}$ are listed in Table 3. Then the off-diagonal van der Waals parameters for each pair of atoms are calculated on the basis of the arithmetic mean. The partial charges for $\mathrm{O} \_\mathrm{z}$ $(-0.19|\mathrm{e}|)$ and $\mathrm{Si} \mathrm{z}(+0.38|\mathrm{e}|)$ are taken from the calculations of Burchart, and those for the atoms in benzene are computed using the AM1 method, available in MOPAC 7.0. ${ }^{23}$

Grand Canonical Monte Carlo Simulation. The GCMC simulation may be the most common technique for predicting the zeolite adsorption phase equilibra from molecular simulations. ${ }^{9,10}$ The GCMC method simulates the equilibrium of a collection of adsorbates in a micropore at constant chemical potential, volume, and temperature or pressure. The bulk pressure can be determined from the chemical potential using a Lennard-Jones equation. So the GCMC simulation technique enables one to study many important characteristics of the fully loaded zeolites under certain pressures and temperatures.

In a GCMC simulation, the initial configuration is generated by one of four moves, for which the acceptance criteria are different. First, a random molecule is picked from the list of sorbates and is placed in a random position and orientation in the framework. The new configuration is accepted with probability $P$

$$
P=\min \left[1 ; \exp \left(-\frac{\Delta E}{k T}-\ln \frac{\left(N_{i}+1\right) k T}{f_{i} V}\right)\right]
$$

where $P$ is the probability of the new configuration being accepted, $\Delta E$ is the energy change between the new configuration and the previous configuration, $k$ is Boltzmann's constant, $T$ is the temperature of the simulation, $N_{i}$ is the current number of molecules of component $i$ in the framework, $f_{i}$ is the fugacity of component $i$ in the gas phase, and $V$ is the cell volume. Second, a molecule is removed from the framework. The simulation first randomly chooses which sorbate type to remove, and then it randomly selects a molecule of that type in the framework. The new configuration is accepted with probability $P$

$$
P=\min \left[1 ; \exp \left(-\frac{\Delta E}{k T}+\ln \frac{N_{i} k T}{f_{i} V}\right)\right]
$$

Third, a sorbate molecule in the framework is chosen at random and translated by a random amount within a cube of size $2 \delta$ (where $\delta$ is the maximum step size). The new configuration is accepted with the probability $P$

$$
P=\min [1 ; \exp (-\Delta E / k T)]
$$

In the fourth type of move, a random sorbate molecule is chosen in the framework. The rotation axis is chosen at random, and the molecule is rotated by a random amount within the range $-\delta$ to $+\delta$. The new configuration, based on the energy change, is accepted with the same probability applied to the translation above.

Eight unit cells of zeolite $(2 \times 2 \times 2)$ are used to construct the simulation box, and periodic boundary conditions are applied. For the real equilibration of the system to be achieved, the length of the simulations must total $6 \times 10^{6}$ steps, and every 600 steps, a configuration of the system must remain. The first 3 million steps are used for equilibration and not included in the averaging. A cutoff of $10 \AA$ is applied to the Lennard-Jones interactions, and the long-range electrostatic interactions are calculated using the Ewald summation technique. The Ewald summation for calculating the adsorbate-adsorbent and adsorbate-adsorbate interaction energies is generally time-consuming. Therefore, a grid-interpolation procedure is used in which the simulation box is split into a collection of small cubes. The grid interpolation method allows taking into account any degree of accuracy in the description of the adsorbate-zeolite and adsorbate-adsorbate interaction energies since all the needed grids are calculated separately prior to any simulation runs. First, GCMC calculations are carried out under standard temperature $(300 \mathrm{~K})$ and standard pressure $(1 \mathrm{~atm})$ to analyze the sorbate location in zeolite at standard condition. Then a series of simulations are performed to predict the adsorption isotherms at $315 \mathrm{~K}$ and $0.0-1.4 \mathrm{kPa}$ pressure. In the first stage of the simulations, the CVFF is used to predict the adsorption isotherms and the distributions of benzene in the zeolite cavities. Then the Burchart-Dreiding force field is applied as a comparison. From our previous work, ${ }^{24}$ it seems that the minor distortions of the ITQ-1 framework did not introduce large effects to the static distributions of benzene, so in GCMC simulations, the atoms of the zeolite framework are fixed at their crystallographic positions - they cannot undergo conformational change.

Molecular Dynamics. MD has become a standard tool in computational chemistry since its introduction in 1957 by Alder and Wainwright. ${ }^{25}$ The technique has been applied to a wide range of systems from molten inonic solids to enzyme-substrate interactions to probe the diffusion mechanism which underpins shape-selective catalysis. The MD method requires a potentialenergy function to describe the energy and first-derivative vector of the system as a function of its structure. Given the forces provided by this energy function and the known atomic masses, we can readily obtain the acceleration vector for the system. A set of starting velocities provides a starting point, and the MD method proceeds by solving Newton's equation of motion using a finite time step. This numerical integration procedure yields a new set of atomic coordinates and forces. Repetition of this algorithm yields a detailed picture of the evolution of the system as a function of time.

In this work, periodic atomistic MD calculations are performed to simulate the diffusion of benzene in ITQ-1. The simulated zeolitic system comprises a $2 \times 2 \times 2$ macrocell of $\mathrm{SiO}_{2}$ with a total of 1728 atoms, to which the periodic boundary condition is applied. The loading simulated is 16 sorbate molecules: eight of them are placed in the 10-MR channels and the other eight placed in the 12-MR supercages. Because 

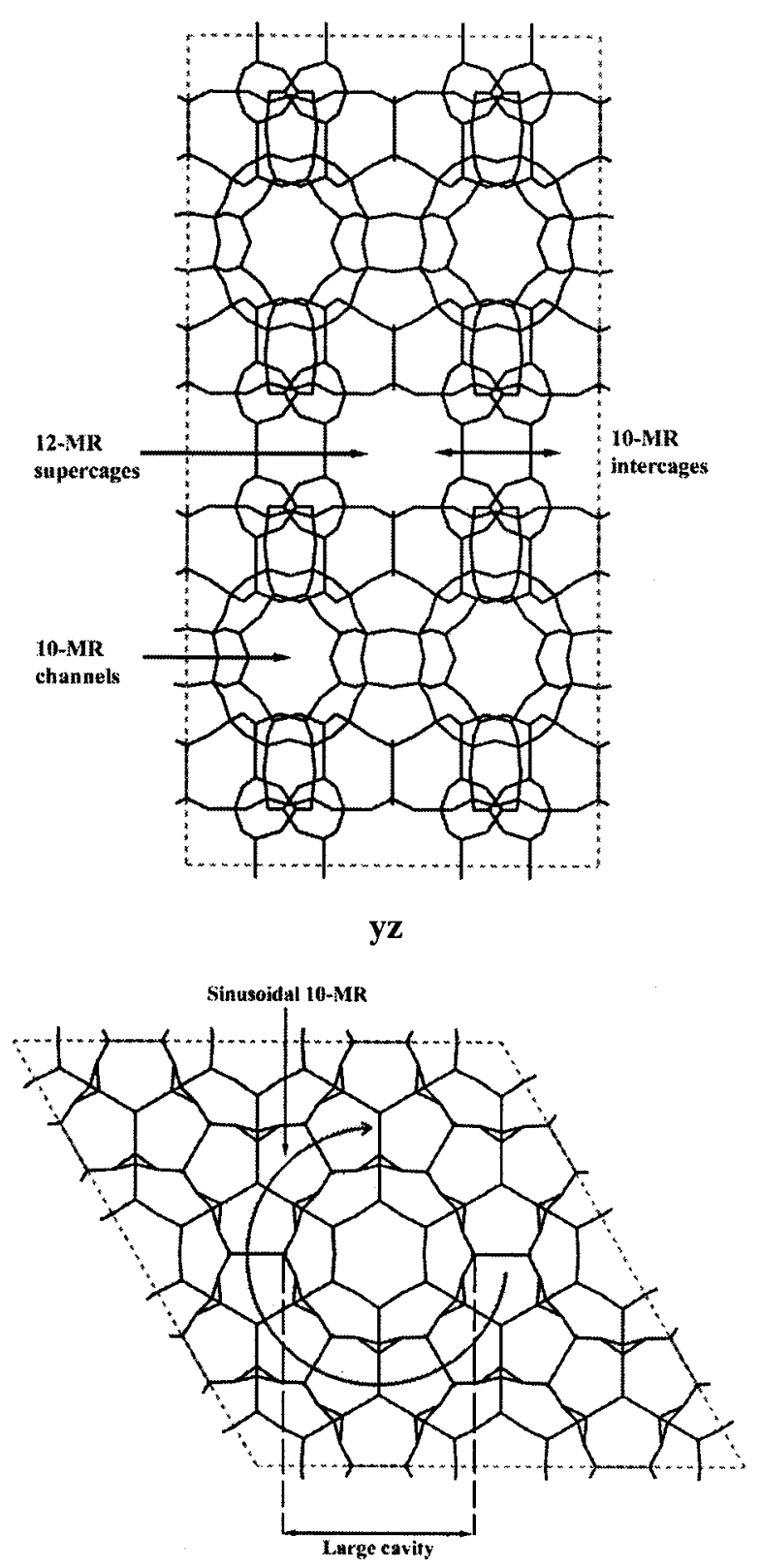

$\mathbf{x y}$

Figure 1. Schematic view of the independent pore systems in ITQ-1. The sinusoidal 10-MR channels are all interconnected to each other, and multiple diffusion trajectories can be allowed to every diffusing molecule. The 12-MR channels are independent of the 10-MR channels, which are interconnected through short 10-MR window systems.

the two channel systems in ITQ-1 are independent, the benzene molecules in both the systems can be studied simultaneously without the need to perform two separate runs, therefore saving much simulation time. Moreover, the studied macrocell includes eight unit cells which contain a complete 12-MR supercage. To explore the interaction between the sorbate molecules, we placed four benzene molecules in this supercage.

The electrostatic interaction is evaluated through the Ewald sum method and van der Waals interactions with a $10 \AA$ cutoff. The simulated system is first optimized at $0 \mathrm{~K}$ using the lattice minimization technique. Then, to ensure that the energy is stationary, we used the minimized result as input for the $25 \mathrm{ps}$ equilibration. After this period, additional runs of 150 ps with a time step of $1 \mathrm{fs}$ are carried out within a NVE microcanonical ensemble at $650 \mathrm{~K}$. We expect that in relatively high temper-

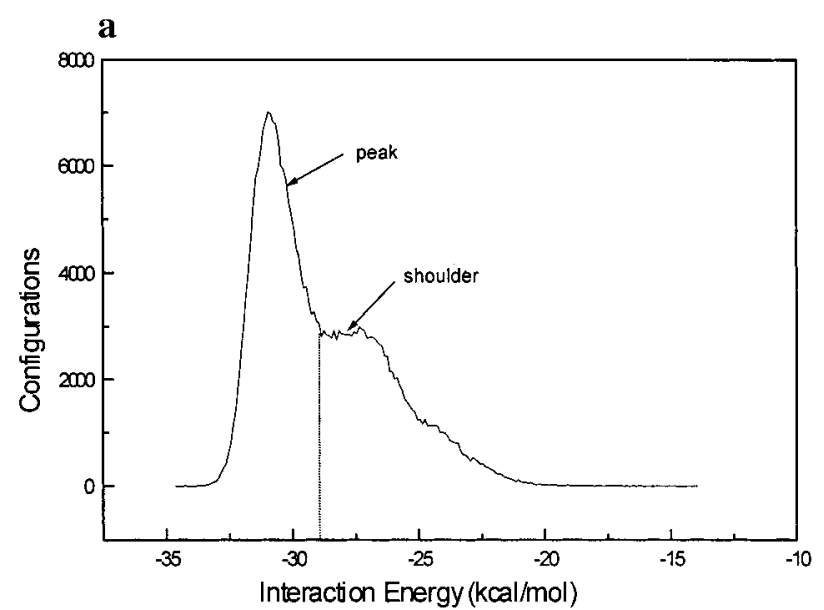

$\mathbf{b}$

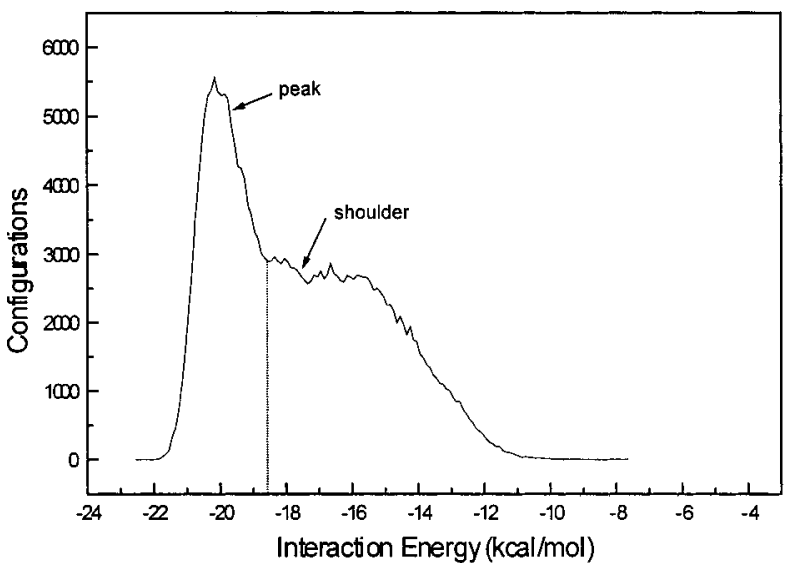

Figure 2. Benzene-zeolite interaction potential energy distribution (a) using the CVFF (b) and the Burchart-Dreiding force field.

atures, the mobility of benzene will be large enough to be investigated on the simulated time scale. The history file from which data analysis of the diffusion process is carried out is saved every $200 \mathrm{fs}$.

\section{Results and Discussion}

A deep insight into the channel systems present in ITQ-1 reveals some special features which will greatly affect the localization and diffusion of benzene in the zeolite lattice (Figure 1). First, all 10-MR sinusoidal channels are interconnected with each other and have high tortuosity. The benzene molecules may be restricted through sinusoidal channels in ITQ-1. Second, the larger 12-MR cavities have large dimensions of $7.1 \AA \times$ 18.2 $\AA$, so the sorbate molecules are expected to host and migrate relatively freely.

Distributions of Benzene in the Two Independent Channel Systems. After $6 \times 10^{6}$ steps of simulations, the equilibration has been achieved, which is indicated from the loading curve and the total energy distribution of the simulated system. At $300 \mathrm{~K}$ and $1 \mathrm{~atm}$, the mean loading of benzene is 69.93 per 8 unit cells. The average interaction energy for every sorbate is about $-29.16 \mathrm{kcal} / \mathrm{mol}$. Figure $2 \mathrm{a}$ depicts the distributions of nonbonding interactions of benzene. The distribution curve is roughly single-peaked, with a maximum around $-31 \mathrm{kcal} / \mathrm{mol}$ and a shoulder from approximately -29.0 to $-26.5 \mathrm{kcal} / \mathrm{mol}$.

To characterize the location of the adsorbed benzene molecules, we have depicted several mass clouds. As a very powerful analysis tool, the mass cloud shows the preferred positions of the adsorbates in zeolite. The mass cloud of benzene 


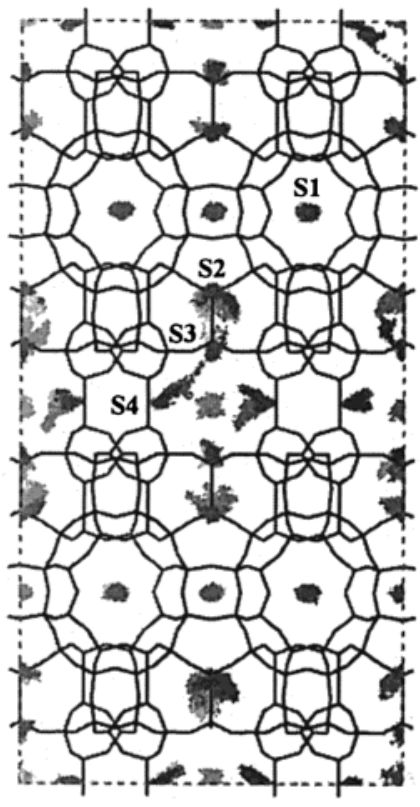

(a)

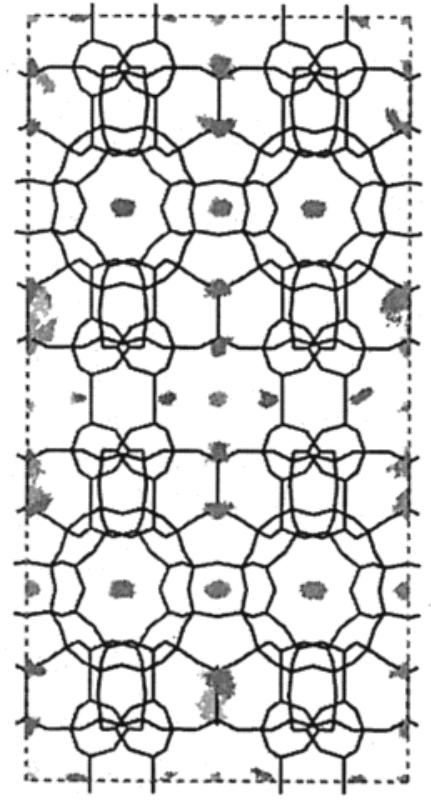

(b)

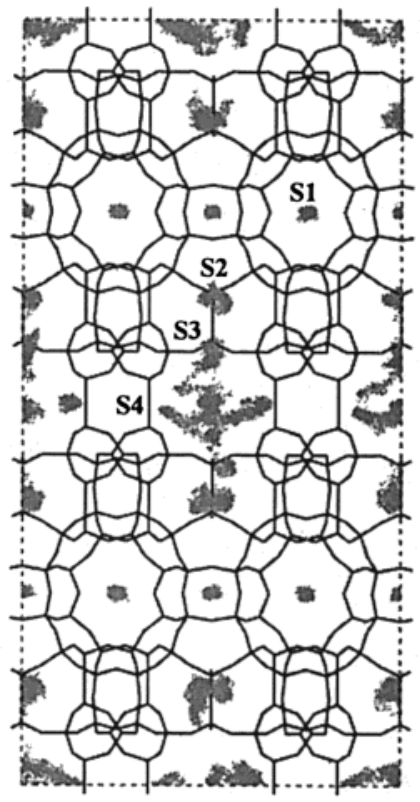

(c)

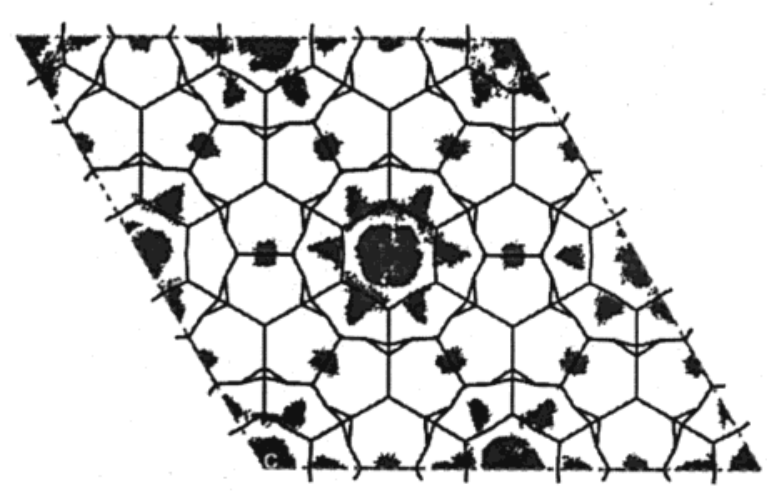

(d)

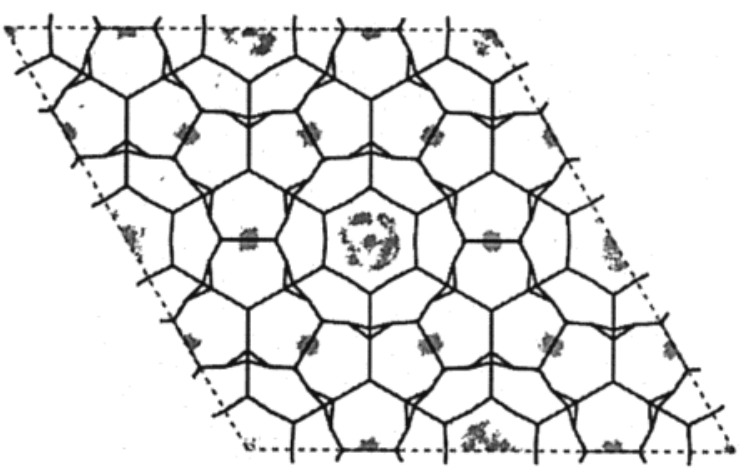

(e)

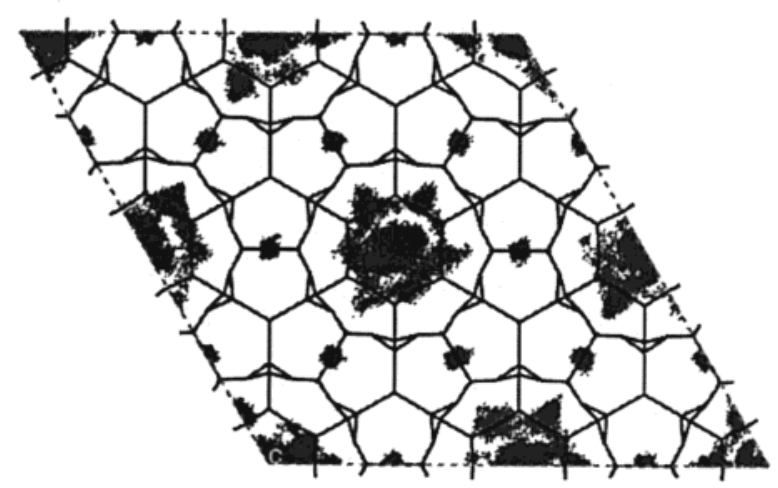

(f)

Figure 3. (a) Mass cloud of benzene in zeolite lattice using the CVFF along the $y z$ direction. (b) Mass cloud of benzene ranging between -100 and $-31.0 \mathrm{~kJ} \mathrm{~mol}^{-1}$ using the CVFF along the $y z$ direction. (c) Mass cloud of benzene using the Burchart-Dreiding force field along the $y z$ direction. (d) Mass cloud of benzene in zeolite lattice using the CVFF along the $x y$ direction. (e) Mass cloud of benzene ranging between -100 and $-31.0 \mathrm{~kJ} \mathrm{~mol}^{-1}$ using the CVFF along the $x y$ direction. (f) Mass cloud of benzene using the Burchart-Dreiding force field along the $x y$ direction.

with respect to the zeolite framework is shown in Figure 3. The center of mass for each sorbate in each configuration is displayed as a dot in the model space. From Figure 3a, it can be observed that the spatial distributions of benzene are roughly territorial and can be divided into four regions: one in the 10-MR channels and three in the 12-MR supercages, S1 and S2-S4, respectively.

The most probable average interaction energy of benzene is around $-31 \mathrm{kcal} / \mathrm{mol}$ (Figure $2 \mathrm{a}$ ). The benzene molecules with interaction energies lower than $-31 \mathrm{~kJ} / \mathrm{mol}$ are presented in Figure $3 b$; it can be seen that many sorbate molecules in the center region of the 12-MR supercages disappeared, indicating that some sorbate molecules located near the center of the supercages are more energetically unfavorable than those near the S1, S2, and S3 sites. Compared with that in Figure 3a, the distribution of benzene near the $\mathrm{S} 1$ site in Figure $3 \mathrm{~b}$ is nearly the same, which allows us to conclude that the sorbate molecules 
near the S1 site have low interaction energies, although they only move within a very restricted area around the minimumenergy position. Benzene molecules can normally diffuse through the 10-MR channel, but in the case of ITQ-1, there is a high degree of tortuosity in the circular channel. Moreover, the circular 10-MR channel in ITQ-1 is much smaller (only 4.0 $\AA \times 5.5 \AA)$ in size than that in ZSM-5 (4.0 $\AA$ × $5.6 \AA$ and 5.1 $\AA \times 5.5 \AA$ for the straight and sinusoidal channels, respectively), which generates the difficulty of diffusion for benzene through the 10-MR sinusoidal channels of ITQ-1. A similar conclusion can be drawn from the dynamics simulations of the 10-MR sinusoidal channels in panel b of Figure 3: the calculated diffusion coefficients are $4.00 \times 10^{-7} \mathrm{~cm}^{2} / \mathrm{s}$ (rigid zeolite framework) and $2.06 \times 10^{-7} \mathrm{~cm}^{2} / \mathrm{s}$ (flexible zeolite framework), which are significantly smaller than the diffusion coefficients of benzene in 12-MR supercages. The benzene molecules are localized at the S2 and S3 sites, where the interaction energy of benzene is relatively low. Site S4 is located near the central part of the supercage, where the molecules of benzene can move in a much wider area. The central part of the supercage possesses a relatively large accommodation space, and the sorbate molecules can stay in the supercages very easily. Moreover, in the 12-MR supercages, the sorbate molecules are energetically acceptable, so the molecules of benzene near S4 are considerably delocalized in the vicinity of their preferred sites of adsorption. Nevertheless, benzene molecules are not observed near the 10MR interconnecting region between two 12-MR supercages, where the benzene molecules are energetically unfavorable.

As a comparison, the same simulations are carried out by using the Burchart-Dreiding force field with the same simulated conditions. At $300 \mathrm{~K}$ and $1 \mathrm{~atm}$, the mean loading of benzene is 62.00 per 8 unit cells. The average interaction energy for every sorbate is about $-17.65 \mathrm{kcal} / \mathrm{mol}$. Figure $2 \mathrm{a}$ depicts the distribution of nonbonding interactions of benzene, which is also roughly single-peaked with a maximum around $-20.2 \mathrm{kcal} /$ mol and a shoulder from approximately -18.6 to $-15.6 \mathrm{kcal} /$ mol. The nonbonding interaction energy for every benzene molecule displays the visible difference between these two force fields. The nonbonding interaction energy from the CVFF is significantly lower than that from the Burchart-Dreiding force field; consequently, the result of the mean loading of benzene using the CVFF is higher than that using the Burchart-Dreiding force field for 8 benzene molecules per 8 unit cells. Figure $3 \mathrm{c}$ shows the mass cloud from simulations using the BurchartDreiding force field. The rough distributions of benzene using these two force fields are similar. The S1, S2, and S3 adsorption sites can also be distinguished very clearly. The most obvious difference is the benzene distribution near the S4 site, where the benzene molecules seem to be able to move more freely when using Burchart-Dreiding force field.

Predicted Isotherms Using Two Different Force Fields. A series of simulations are performed to get the adsorption isotherms. For comparison with some experimental results, ${ }^{26}$ the simulations are set at a temperature of $315 \mathrm{~K}$ and some pressures ranging between 0.0 and $1.4 \mathrm{kPa}$. The calculated adsorption isotherms of benzene in ITQ-1 at $315 \mathrm{~K}$ using two different force fields are shown in Figure 4. Because the adsorption isotherms of benzene from experiments are unavailable, the experimental isotherm of toluene is used as a comparison. Certainly the isotherms of benzene and toluene cannot be quantitatively compared, but their structures are similar, so their interactions with the zeolite framework and adsorption behaviors should be similar. Only from the viewpoint of volume is benzene smaller than toluene, so its adsorbed

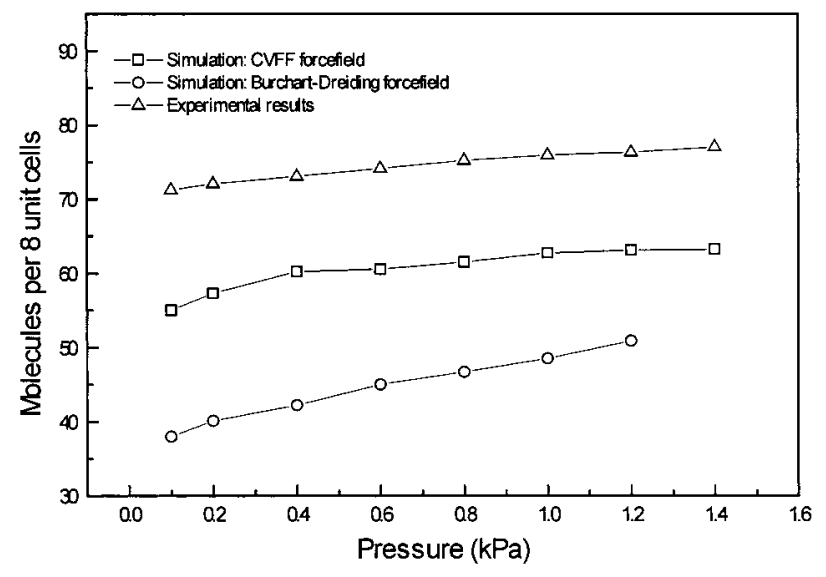

Figure 4. Simulated adsorption isotherms of benzene using the two different force fields at $315 \mathrm{~K}$ and experimental values for toluene.

amount in ITQ-1 may be larger than that of toluene, which can be well indicated from both the predicted isotherms using two different force fields (Figure 4). Previous experiments of a combined adsorption-microcalorimetric study have been applied to explore the adsorption-diffusion behaviors of toluene, $m$ - and $o$-xylene, and 1,2,4-trimethylbenzene with different kinetic diameters in MCM-22, ${ }^{25}$ and the adsorption isotherms have validated that the zeolite uptake relies significantly on the size of the adsorbate molecules. It can be observed that the uptake of $m$-xylene is about half that of toluene. The value for $o$-xylene is much lower and approximately the same as that for 1,2,4-trimethylbenzene. From our simulations, it can be noted that the uptake of benzene is much higher than the value of toluene, which is also preferably in accord with previous researches.

From the predicted isotherms, the diffusion of benzene is found to happen at very low pressure. When the pressure is below $0.1 \mathrm{kPa}$, the loading of benzene increases rapidly from 0 at $0.0 \mathrm{kPa}$ to a certain amount at $0.1 \mathrm{kPa}$, but the increase becomes relatively slow as the pressure progresses higher than $0.1 \mathrm{kPa}$. The reason is the favorable interactions between the sorbate molecules and the zeolite framework, which makes benzene possess relatively high loading at low pressure. But from two predicted isotherms using two different force fields, it is very clear that these two force fields yield relatively different results. The calculated interaction between benzene and zeolite framework using the CVFF is obviously weaker than that using the Burchart-Dreiding force field, which results in the uptake of benzene using the CVFF being higher than that using the Burchart-Dreiding force field. In the GCMC simulations, the zeolite framework is assumed to be rigid, and the potential of the system only comes from van der Waals and coulomb interactions. For these two sets of potential parameters, the coulomb interactions do not introduce obvious differences since the partial charges of these two force fields do not differ greatly, so the simulated difference mainly comes from the different van der Waals parameters. But until now, it has been very difficult for us to determine which force field is better. Even if we have some experimental results, there are still problems for getting decisive results because there exists some difference between the realistic experiments and the simulated model. Nevertheless, our simulated results reveal that the two force fields bring obvious differences, and the force field should be carefully chosen for the zeolite simulations.

Diffusivity in Flexible vs Frozen Framework. The MD simulations are used to explore the dynamic diffusion behavior of the benzene molecules in ITQ-1. In this stage, the potential 
parameters from CVFFs are used, including complete integrated potential parameters for nonbonding and covalent interactions. Using this force field, we can fully explore the influence of the framework and the sorbate molecules. It is well-known when the guest molecules diffuse or migrate inside the cavities of zeolites to get the best energetic and shape complementarity between the zeolite and the sorbate molecules, and the conformation of the zeolite framework should be changed to a certain degree. But for different zeolites and sorbates, the influence of the framework flexibility is quite different. So in this paper, to investigate the influence of framework flexibility on the diffusivity of the guest molecule, we carried out the MD of the diffusion of benzene in flexible and rigid frameworks. During the simulation, the trajectory of the system is saved every 200 fs, and subsequently, the mean-square displacements (MSD) of the guest molecules can be calculated

$$
\left\langle X^{2}(t)\right\rangle=\frac{1}{N_{m} N_{t_{0}}} \sum_{m} \sum_{t_{0}}\left[X_{i}\left(t+t_{0}\right)-X_{i}\left(t_{0}\right)\right]^{2}
$$

where $N_{m}$ is the number of diffusing molecules, $N_{t_{0}}$ is the number of time origins used in calculating the average, and $X_{i}$ is the coordinate for the center of mass of molecule $i$.

Figure 5 shows the MSD plot for benzene in flexible and frozen frameworks. The plots in different channel systems show that the MSD for benzene in 12-MR supercages is significantly larger than that in 10-MR sinusoidal channels, which means that the mobility of benzene in 10-MR channel systems is greatly restricted. But the comparison of MSD for benzene in the flexible and frozen frameworks does not present noticeable differences. The flexibility of the framework does not have great effects on the diffusivity or mobility of benzene.

After obtaining the MSD of benzene, we can compute the diffusion coefficients, $D$, using the Einstein relation

$$
\left\langle X^{2}(t)\right\rangle=6 D t+B
$$

where $t$ is the simulation time and $B$ is the thermal factor arising from atomic vibrations. The diffusion coefficients of benzene in two independent channel systems are listed in Table 4, where it can be found that there are not noticeable differences between the results from flexible and rigid frameworks. The MD of Sastre's work gave diffusion coefficients of benzene in 10-MR sinusoidal channels and 12-MR supercages, $6.60 \times 10^{-7}$ and $4.62 \times 10^{-6} \mathrm{~cm}^{2} / \mathrm{s}$, respectively, ${ }^{27}$ which were a little larger than those values from our simulations. On the order of magnitude, there does not exist obvious difference. We think that the difference mainly comes from the usage of different potential parameters. Considering that the zeolite framework undergoes certain conformational flexibility in actual adsorption process, the following discussions will only refer to the simulated results using the flexible framework.

Trajectories of Benzene in the 12-MR Supercages. The mass cloud from Monte Carlo simulations, the calculated MSD, and the diffusion coefficient of benzene in different channel systems have shown that the diffusion and the mobility of benzene mainly occur in 12-MR supercages. Figure 6 a shows the four benzene molecules in the complete 12-MR supercage after 35 ps of equilibration of MD. It can be found that two molecules are distributed near the center of the supercage, while the other two are located near the upper and lower parts of the supercage with relatively small space. To clarify the diffusivity of benzene more visually, we projected the trajectories of the motion of benzene on the framework. In Figure 7 are displayed
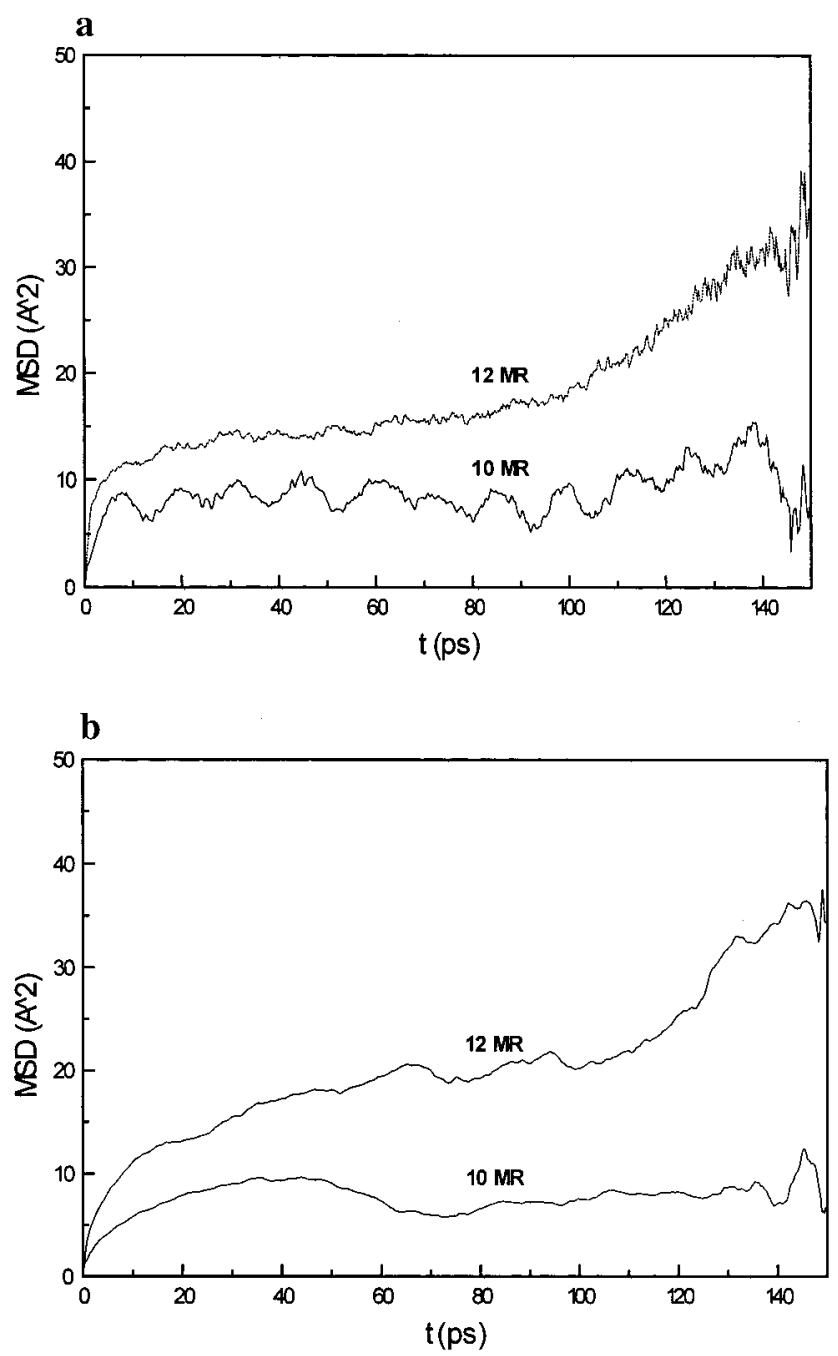

Figure 5. Mean-square displacements (MSD) for the 150 ps runs of benzene in the 10-MR sinusoidal system and the 12-MR supercage system of (a) the frozen framework and (b) the flexible framework. The diffusion coefficients of benzene, obtained from eq 9, are shown in Table 4.

TABLE 4: Diffusion Coefficient of Benzene in the Zeolitic Framework

\begin{tabular}{lcc}
\hline & \multicolumn{2}{c}{ diffusion coefficients $\left(\mathrm{cm}^{2} / \mathrm{s}\right)$} \\
\cline { 2 - 3 } & $10 \mathrm{MR}$ & $12 \mathrm{MR}$ \\
\hline frozen framework & $4.00 \times 10^{-7}$ & $2.32 \times 10^{-6}$ \\
flexible framework & $2.06 \times 10^{-7}$ & $2.56 \times 10^{-6}$
\end{tabular}

the trajectories followed by these molecules in the 12-MR supercage. Figure 7 a shows the trajectories of molecules 1 and 2 , which possess relatively good diffusivities and large motion spaces. The projection of benzene along the $y z$ orientation indicates that molecules 1 and 2 spend more time in two regions: the first one is located near the central part of the supercage, while the other one is near the S2 site in Figure 3. Figure $7 \mathrm{~b}$ presents the projection of the four molecules in Figure $6 \mathrm{a}$ on the framework along the $y z$ orientation. Compared with molecules 1 and 2, molecules 3 and 4 possess quite different characteristics of motions, and they are almost restricted to a very small area (S2 site in Figure 3) around the minimum energy position. The trajectories of benzene in 12-MR supercages are quite similar to the distribution from the mass cloud of benzene in Figure 3.

Two neighboring 12-MR supercages are connected with the 10-MR windows system, so intercage motion is, in principle, 
$\mathbf{a}$
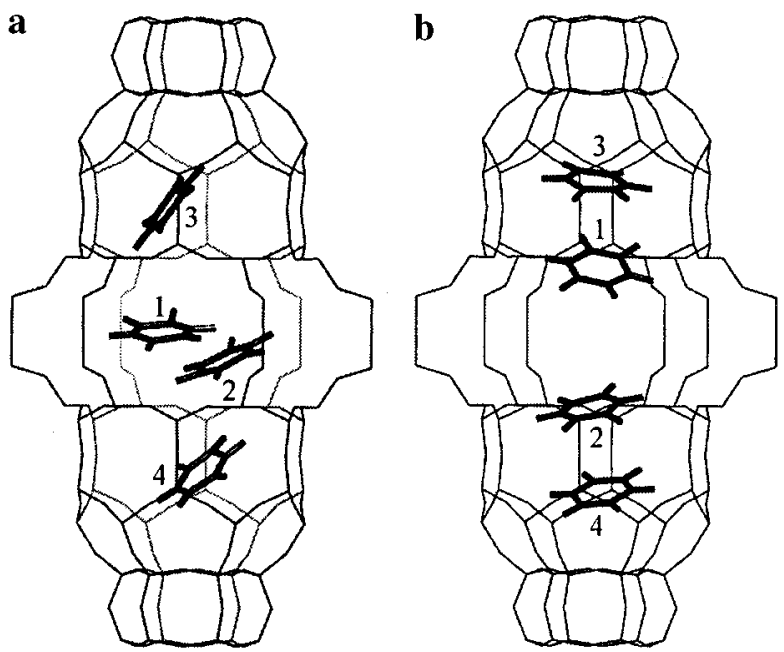

Figure 6. The distribution of four benzene molecules in one 12-MR supercage: (a) the conformation after 35 ps and (b) 120 ps of MD equilibrium.

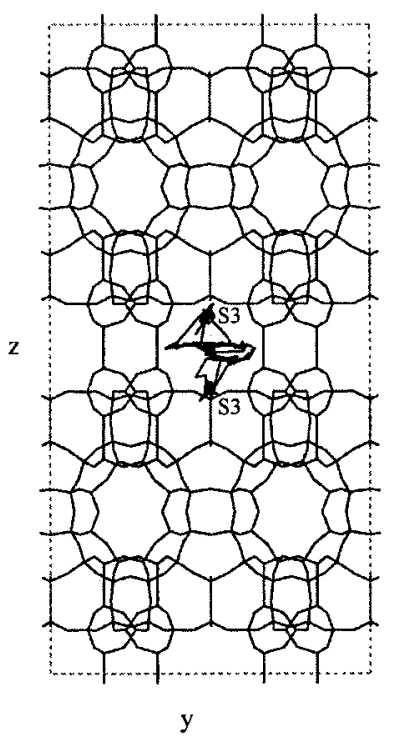

(a)

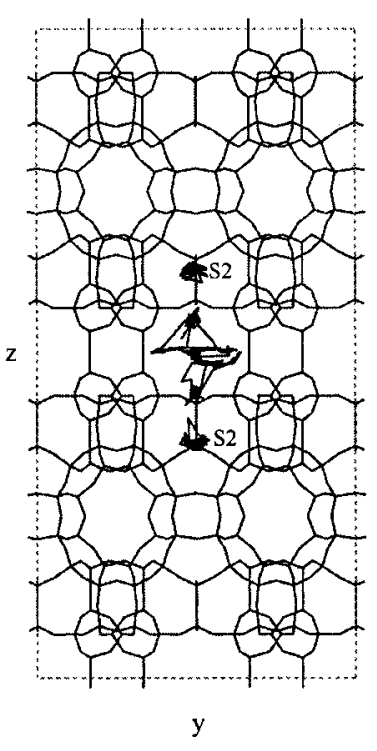

(b)
Figure 7. (a) Trajectories of molecules 1 and 2 in the 12-MR supercage. (b) Projections of the four molecules in Figure 6a on the framework along the $y z$ orientation.

possible. Nevertheless, migration of benzene molecules from one supercage to another has not been observed in this simulation, and this is in part due to the size and position of the 10-MR interconnecting windows, which are perpendicular to the direction of the motion, and then the interconnecting motion is not energetically favorable.

In this paper, the adsorption isotherms were predicted in section 2, and the trend of loading for benzene seems to agree with the results of experiments on aromatics. From the principle of GCMC, it can be seen that this technique adds the sorbate molecules to the cavities or micropores in the zeolite framework using Metropolis criteria and only tests the final adsorption equilibria in zeolite without considering the energetic states along the adsorbate diffusion pathway. So if there exist some potential barriers while adsorbate migrates through the channel systems, the adsorbate molecules may not migrate into the interior cavities or micropores in the zeolite, and the resulting GCMC calculations will overestimate the adsorbed amount of adsorbate. But the predicted values of benzene seem to be consistent with previous experimental results, which means that
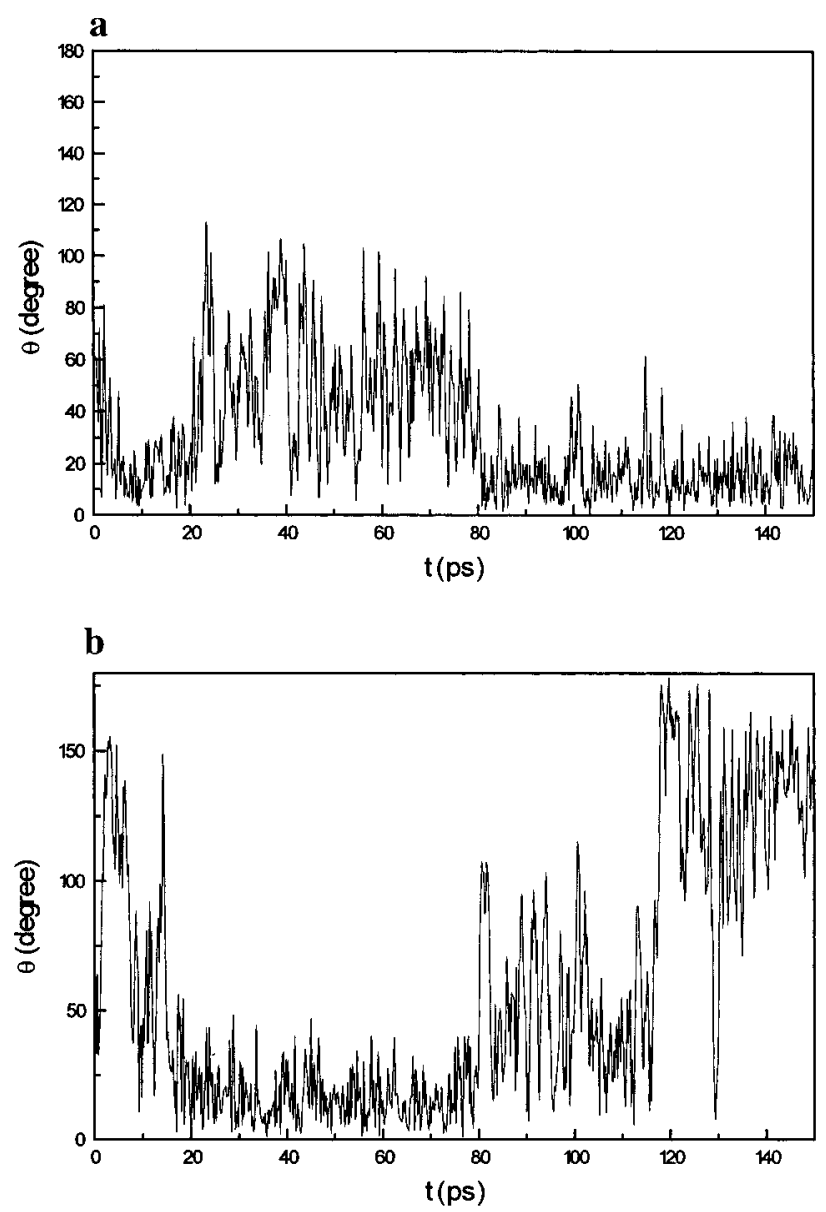

Figure 8. Fluctuation of the angle defined by (a) benzenes 1 and 3 (b) and benzenes 2 and 4 .

during the diffusing process, the benzene molecules do not encounter obvious potential barriers. From the results of MD in this paper and previous work by Sastre et al., ${ }^{26}$ the intercage motions of benzene are not observed at $615 \mathrm{~K}$, which suggests that 150 ps MD in this paper and 200 ps MD in Sastre's work are too short to obtain the interconnecting migrations of benzene. So the diffusion coefficient of benzene in this paper is only a rough estimate for benzene intracage motions.

Interactions among Sorbate Molecules. In Figure 6, four benzene molecules are placed in the complete supercage. The diffusivity of benzene is affected not only by the interactions between sorbates and framework but also the interactions between the sorbate molecules, especially at high loading. Figure $7 \mathrm{a}$ indicates that molecules 1 and 2 spend relatively long times near S3 sites. Moreover, from the dynamic trajectory of benzene, it can be found that molecules 1 and 3 or 2 and 4 exhibit some motion regularities. The change of the angle between the planes of molecules 1 and 3 or 2 and 4 (see Figure 8) is investigated during the simulations. It is very clear that when molecules 1 and 2 migrate to near the S2 site, the molecular planes of sorbates 1 and 3 or 2 and 4 will adopt a parallel state. Molecules 1 and 2 will spend half the simulation time (about 75 ps) near the $\mathrm{S} 3$ sites, where the motions between two benzene molecules near S1 and S2 sites will keep a very synergistic status to maintain the the system's minimum energetic state.

When molecules 1 and 3 or 2 and 4 become parallel, the distance between their mass centers is about $4-5 \AA$, and the pair of parallel molecular planes of benzene should produce relatively strong aromatic stacking interactions. The energetic states of molecules 1 and 3 or 2 and 4 are investigated when 
they become parallel. Figure 8 shows the conformation of the system at $120 \mathrm{ps}$, when molecules 1 and 3 or 2 and 4 just become nearly parallel. First, the conformation at $120 \mathrm{ps}$ is minimized by using molecular mechanics to obtain a more reasonable structure. After minimization, the total energy of molecule 2 or 4 is computed to be 6.840 or $6.272 \mathrm{kcal} / \mathrm{mol}$, respectively, which comes from two parts, including the selfcovalent potential of the sorbate molecules and the nonbonding interactions between molecule 2 or 4 and the zeolite framework. When molecules 2 and 4 become parallel, they will bear similar energetic states, but molecule 4 seems to be somewhat more energetically favorable. Then molecule 2 is taken from the system, and afterward, the system is fully minimized. Without molecule 2, the total energy of molecule 4 is about $10.258 \mathrm{kcal} /$ mol. In the same way, molecule 2 is investigated without molecule 4, and the total energy of molecule 2 is found to be about $10.258 \mathrm{kcal} / \mathrm{mol}$. It is very clear that when molecules 2 and 4 do not exhibit mutual interactions, their energetic states are quite different, and the total energy of benzene 4 is $1.1 \mathrm{kcal} /$ mol higher than that of benzene 2 . If only the single molecule 2 or 4 is considered, the sorbate molecules near the S2 site are more energetically favorable. But when molecule 2 moves near the S2 site and becomes parallel with molecule 4, the nonbonding interactions will reduce their energies significantly. For molecules 2 and 4, the reduced energies are 4.4 and $5.1 \mathrm{kcal} /$ mol, respectively. It can be concluded that the nonbonding interactions between the sorbate molecules will have a large influence on the diffusivity and mobility of the sorbate molecules. It can be deduced that the existence of the $\mathrm{S} 2$ site is the synergistic result for the sorbate molecules.

\section{Conclusions}

Molecular simulations of the location and diffusion of benzene in ITQ-1 are presented. The simulations are performed using GCMC simulations and MD. The combination of these two techniques provides enough static and dynamic information about the diffusion of benzene in zeolite cavities, which is very helpful for us to interpret the shape selectivity and reactive processes in ITQ-1. From these simulations, a coherent picture of the adsorption of benzene in ITQ-1 can be drawn, including the static distributions of benzene and the dynamical migration process in 10-MR sinusoidal channels and 12-MR supercages.

The mass clouds of benzene from GCMC simulations show that the distributions of benzene in the 10-MR channel are restricted to a very small region, while the distributions in 12MR supercages are dispersed in a relatively large area, which means that the diffusion and mobility of benzene mainly happened in 12-MR supercages. Moreover, the distributions of benzene in 12-MR supercages can be distinctively separated into three adsorption sites, and the sorbate molecules in these sites represent different energetic states. In GMCM simulations, two different force fields, including the CVFF and the BurchartDreiding force field, are applied. Under the same simulated conditions, these two force fields can generally produce similar and consistent results, although the obtained interaction energy is quite different, which is determined by the inherent characteristics of two different force fields.

The dynamic diffusion of benzene in the zeolite is analyzed by using MD. The trajectories of benzene are very similar to the mass clouds from GCMC simulations. In the same supercage, the benzene molecules migrate widely in the central region of framework but only locally near the S2 and S3 sites. Moreover, the diffusing characteristics of the benzene molecules indicate that the motions between two benzene molecules near the S2 and S3 sites will keep a very synergistic state, and the pair of benzene molecules in the S1 and S2 sites can produce relatively strong aromatic stacking interactions.

Acknowledgment. The authors acknowledge financial support from the Research Institute of Petrochemical Processing of SINOPEC and NCSF (29992590-2).

\section{References and Notes}

(1) Horsley, J. A.; Fellmann, J. D.; Derouane, E. G.; Freeman, C. M. J. Catal. 1994, 147, 231. 12345

(2) Klein, H.; Kirschhock, C.; Fuess, H. J. Phys. Chem. 1994, 98,

(3) Demontis, P.; Suffritti, G. B.; Alberti, A.; Quartieri, S.; Fois, E. S.; Gamba, A. Gazz. Chim. Ital. 1986, 116, 459.

(4) Yashonath, S. Chem. Phys. Lett. 1991, 177, 54.

(5) Yashonath, S.; Demontis, P.; Klein, M. L. J. Phys. Chem. 1991, $95,5881$.

(6) Catlow, C. R. A.; Freeman, C. M.; Vessal, B.; Tomlinson, S. M.; Leslie, M. J. Chem. Soc., Faraday Trans. 1991, 87, 1947.

(7) Demontis, P.; Suffritti, G. B.; Fois, E. S.; Quartieri, S. J. Phys. Chem. 1992, 96, 1482.

(8) June, R. L.; Bell, A. T.; Theodorou, D. N. J. Phys. Chem. 1992, 96, 1051.

(9) Randall, Q. S.; Alexis, T. B.; Doros, N. T. J. Phys. Chem. 1993, 97, 13742

(10) Van Tassel, P. A.; Davis, H. T.; Mccormick, A. V. J. Chem. Phys. 1993, 98, 4173.

(11) Leonowicz, M. E.; Lawton, J. A.; Lawton, S. L.; Rubin, M. K. Science 1994, 264, 1910.

(12) Rubin, M. K.; Chu, P. U.S. Patent 4954325, 1990.

(13) Wu, P.; Komatsu, T.; Yashima, T. Microporous Mesoporous Mater. 1998, 22, 343.

(14) Asensi, M. A.; Corma, A.; Martinez, A. J. Catal. 1996, 158, 561.

(15) Corma, A. Microporous Mesoporous Mater. 1998, 21, 487.

(16) Cerius ${ }^{2}$ Simulation Tools User Reference; Molecular Simulation Inc.: San Diego, CA, 1998.

(17) Yang, P. C.; Ye, X. Q.; Ji, M. J.; Zhao, Z. L.; Zhang, Z. Y.; Hou, T. J.; Xu, X. J. Acta Phys. Chim. Sin. 1998, 14, 686.

(18) Camblor, M. A.; Corma, A.; Baerlocher, C. J. Phys. Chem. B 1998, 102,44 .

(19) Kitson, D. H.; Hagler, A. T. Biochemistry 1988, 27, 7176

(20) Dauber-Osguthorpe, P.; Roberts, V. A.; Osguthorpe, D. J.; Wolff, J.; Genest, M.; Hagler, A. T. Proteins: Struct., Funct., Genet.1988, 4, 31.

(21) Burchart, E. D. V. Ph.D. Thesis, Technische Universiteit Te Delft, 1992.

(22) Mayo, S. L.; Olafson, B. D.; Goddard, W. A. J. Phys. Chem. 1990, 94, 8897.

(23) MOPAC 7.0 User Guide, Quantum Chemistry Program Exchange (OCPE); Indiana University: Bloomington, IN, 1993.

(24) Hou, T. J.; Zhu, L. L.; Xu, X. J. J. Mol. Struct., in press.

(25) Alder, B. J.; Wainwright, T. E. J. Chem. Phys. 1957, 27, 1208

(26) Corma, A.; Corell, C.; Pérez-Pariente, J.; Guil, J. M.; Guil-López, R.; Nicolopoulos, S.; Gonzalez Calbet, J.; Vallet-Regi, M. Zeolite 1996, 16,7 .

(27) Sastre, G.; Catlow, C.; Corma, A. J. Phy. Chem. B 1999, 103, 5187. 